\title{
Plasma cell infiltrates in paediatric renal transplant biopsies are associated with increased risk of renal allograft failure
}

\author{
Stephanie Dufek ${ }^{1}$, Azaz Khalil ${ }^{1}$, Nizam Mamode ${ }^{2}$, Neil J Sebire ${ }^{1}$, Stephen D \\ Marks $^{1}$ \\ ${ }^{1}$ Department of Paediatric Nephrology, Great Ormond Street Hospital for Children NHS \\ Foundation Trust, London, UK \\ ${ }^{2}$ Guy and St Thomas Hospital, NHS Foundation Trust, London, UK
}

Running Title:

Word Count 2524

Abstract 314

References 9

Corresponding author:

Dr Stephen Marks

Consultant Paediatric Nephrologist

Great Ormond Street Hospital for Children NHS Foundation Trust

London WC1N 3JH

United Kingdom

tel: +44 2074059200

fax:+44 2078298841

E-mail: Stephen.Marks@gosh.nhs.uk 


\section{Abstract}

Introduction: Prominent plasma cell infiltration in renal allograft biopsies is a rare finding which is associated with poor outcome in adult renal transplant recipients. The clinical impact of plasma cell rich acute rejection episodes in paediatric renal transplant recipients (pRTR) remains unknown. We investigated the frequency and prognosis in pRTR in a retrospective case-control study.

Materials and methods: We conducted a retrospective study of plasma cell rich acute rejection episodes in pRTR from April 1996 to March 2014. Cases were defined as all pRTR with prominent $(\geq 10 \%$ of infiltrate) plasma cells in renal transplant biopsies. Cases were compared to controls with typical rejection without significant plasma cell infiltration. Cases were matched to control cohort for grade of rejection according to Banff classification, age at Tx and Tx age at biopsy.

Results: Plasma cell infiltrates were present in 14 of 162 (9\%) pRTR, aged 3.2 - 17.5 (median 13.4) years at time of transplantation, of whom $13(93 \%)$ received deceased donor renal transplants. Compared to 14 matched pRTR renal transplant biopsies without significant plasma cells, there were no significant differences in type of Tx (LRD vs DD), mismatch, CMV/EBV status of recipient and patient ????? at Tx and baseline eGFR. Plasma cells were present in case biopsies with a maximal density of 14 - 116 (median 33) plasma cells/hpf/x40. Plasma cells were associated with decreased eGFR at biopsy (22 vs $49 \mathrm{mls} / \mathrm{min} / 1.73 \mathrm{~m} 2 ; \mathrm{p}<0.001$ ), despite comparable eGFR four weeks prior to biopsy. Mean eGFR post biopsy was significantly lower in patients with plasma cells (25.8 vs $55.7 \mathrm{mls} / \mathrm{min} / 1.73 \mathrm{~m} 2 ; \mathrm{p}<0.001)$. Plasma cells were associated with significantly increased frequency of renal allograft loss $(71 \%$ vs $7 \% ; p<0.001)$ at $0-27$ (median 2) months after biopsy. 
Conclusion: Plasma cell rich acute rejection episodes in pRTR are uncommon but associated with reduced renal allograft survival. In the future, bortezomib, a proteasome inhibitor which induces apoptosis in mature plasma cells, may play a role in patient management.

\section{Introduction}

The treatment and outcome of renal allograft acute rejection episodes in adults and children are mainly determined by the histopathological findings according to revised Banff classifications. Acute cellular rejection episodes are classified according to three main criteria: vasculitis/endothelialitis, tubulitis and interstitial infiltration (1). Interstitial infiltrates are mainly composed of $\mathrm{T}$ lymphocytes, monocytes and macrophages. Typically, plasma cells account for $<5 \%$ of infiltrating cells in acute rejection episodes (2). During the last decade, so called plasma cell rich acute rejection (PCAR) has been reported, referring to acute rejection episodes in which plasma cells account for $>10 \%$ of the graft infiltrating cells. To date PCAR has been observed in around $2 \%$ of adults with biopsy proven acute rejection (3) (2) and has been reported to be associated with poor prognosis compared with standard acute rejection with equivalent Banff scoring $(4,5)$.

The frequency and prognostic significance of plasma cell rich acute rejection in paediatric renal transplant recipients remains unknown.

We conducted a retrospective case control study of paediatric renal transplant recipients with acute rejection episodes, comparing those with plasma cell rich infiltrates with standard acute rejection matched for equivalent Banff scoring. 


\section{Materials and methods}

Cases were defined as paediatric renal transplant recipients that underwent a renal allograft biopsy for acute, chronic or acute on chronic allograft dysfunction frrm April 1996 to March 2014 who were found to have subjectively prominent plasma cell infiltration noted on the transplant biopsy (indicating plasma cells $>10 \%$ of the graft infiltrating cells). Cases were matched to a control cohort of paediatric renal transplant recipients who also underwent renal allograft biopsy for the same indications during the same time period at the cntre without significant plasma cell infiltration. Cases were matched to control cohort for age at Tx, type of Tx, patient age at biopsy and Tx age at biopsy and biopsy score according to the revised Banff classification (2007). Patients underwent percutaneous renal biopsy for acute allograft dysfunction as demonstrated by acute rise in plasma creatinine $\geq 10 \%$ from baseline, or chronic renal allograft dysfunction with slowly rising creatinine.

We retrospectively collected demographic, clinical data and previous investigation results from clinical records. Data obtained included the following: sex of patient, primary diagnosis, number of transplants, age at transplant and age at biopsy, age of transplant at biopsy, type of transplantation (deceased versus living related donor), mismatch, EBV and CMV of recipient and donor at time of transplant, renal biopsy findings, presence of donor specific antibodies. Plasma creatinine and eGFR using the Schwartz formula were collected for following time points in regards to the biopsy date: baseline (lowest creatinine reached post transplantation), 3 months prior, 4 weeks prior, date of biopsy and 4 weeks post biopsy. Data on outcome at final follow up were collected.

Graft loss was defined the requirement of renal replacement therapy.

The revised Banff 2007 working classification of renal allograft pathology were used to define AR and Chronic changes (1).

AR was graded as I, II, III or borderline "suspicious" for AR with Grade I AR referring to the histological finding of interstitial lymphocytic infiltration and tubulitits, Grade II AR with 
endothelialitis, Grade III AR with transmural arteritis and/or arterial fibrinoid change, and borderline "suspicious" of AR referring with foci of mild tubulits only Chronic changes of interstitial fibrosis, tubular atrophy, chronic large vessel changes and arteriolar hyalinosiswere noted. Cases with subjectively prominent plasma cell infiltration in the report were identified from the records and matched cases reported with similar Banff severity were identified. All biopsies were then reviewed blindly and maximal plasma cell counts per high power filed (x40 objective) in the most affected areas, were derived. Plasma cells were rare in the controls whereas in the cases numerous plasma cells were present, consistent with the previously suggested criteria of $>10 \%$ of infiltrating inflammatory cells in the region.

\section{Immunosuppression protocol}

Patients in receipt of their first transplant with no history of cytotoxic antibodies received triple immunosuppressive therapy with prednisolone, azathioprine or mycophenolate mofetil and tacrolimus or ciclosporin as their baseline immunosuppression. There was one patient in the control cohort who received asecond deceased donor allograft. This patient received quadruple immunosuppressive therapy including the above triple therapy and additional treatment with basiliximab. Patients with biopsy proven CAN had azathioprine switched to MMF and the calcineurin inhibitor withdrawn or reduced. 


\section{Statistics}

All statistics were performed using SPSS version 22 for Mac. Kaplan-Meier survival curves were used to examine renal allograft survival for those with and without plasma cell infiltrates. Other relevant clinical and laboratory data were compared between cases and control cohort using the Mann-Whitney U-test, or the Fishers exact test with significance set at $p<0.05$. The log-rank test was used for significance testing for the Kaplan-Meier plots of renal allograft survival. Ethical approval for was obtained as per local requirements. 


\section{Results}

\section{General demographics of cases and controls}

Prominent plasma cell infiltrates were present in renal transplant biopsies studied from 14 of $162(9 \%)$ renal transplant recipients who underwent renal transplant biopsies for investigation of acute or chronic allograft dysfunction. Renal transplant recipients with plasma cells present were eight male $(57 \%)$, median age $13.4(3.2-17.45)$ years at time of transplantation, of whom $13(93 \%)$ received deceased donor renal transplants and all had received their first transplant.

The matched control cohort of 14 renal transplant recipients without increased plasma cells were eight male $(57 \%)$, median age $12.2(3.2$ - 16.6) years at time of transplantation of whom nine (64\%) received deceased donor renal transplants and one was re-transplanted.

The primary pathologies of both groups were typical of paediatric end stage renal failure and are summarized in Table 1.

There were no significant differences between cases and controls in age at transplant, sex (type of transplant (LRD vs DD), mismatch, CMV/EBV status of recipient at TX and patient and TX age at biopsy and baseline eGFR in $\mathrm{mls} / \mathrm{min} / 1.73 \mathrm{~m}^{2}$ (Table 2).

\section{Immunosuppression}

Baseline immunosuppression in cases consisted of Prednisolone, Tacrolimus, Azathioprine $(n=9)$ or Prednisolone, Tacrolimus, Mycophenolate Mofetile (MMF) ( $n=2)$ or Prednisolone, Ciclosporin, Azathioprine $(n=2)$ or Prednisolone, Tacrolimus, Rituximab in 1 patient who had a previous heart and lung transplantation. Within the controls baseline immunosuppression was Prednisolone, Tacrolimus, Azathioprine $(n=9)$ or Prednisolone, Tacrolimus, Mycophenolate Mofetile $(n=1)$ or Prednisolone, Ciclosporin, Azathioprine $(n=1)$ or Prednisolone, Tacrolimus, MMF, Basiliximab $(n=1)$ or Prednisolone, Tacrolimus, MMF, Dacluzimab $(n=1)$ or Prednisolone, Tacrolimus, Azathioprine and iv IG in the one patient who received the second transplant. 


\section{Plasma cells and biopsy findings}

The median age of cases at biopsy was $15.9(4.9$ - 17.9) years, with a transplant age of median 18 (4.9 - 106.5) months compared to median age at biopsy of 13.5 (5.6 - 17.6) years and median transplant age of 8.7 (0.7 - 103.2) months for controls (STATS??). There was no significant difference in number and grade of rejection according to Banff classification between cases and controls. In the group with plasma cells one patient had plasma cells only and one patient had findings of tubuluinterstitial nephritis, which could not be classified within the BANFF criteria (Table 3).

Plasma cells were present in cases with a median maximal density of 46 (range $14-116$ ) plasma cells/hpf. Donor specific antibodies were present in 7 (50\%) patients (57\% Class I, $29 \%$ Class II and $14 \%$ Class I and II HLA antibodies) with $57 \%$ of them having increased titres giving total MFI of $1252-7709$.

\section{Plasma cells and allograft function}

There was no significant difference in eGFR at baseline (75.9 vs 88.3; $p=0.175$ ), three months prior to biopsy (59.3 vs $76.1, p=0.79$ ) and four weeks prior to biopsy (48.9 vs 63.7; $\mathrm{p}=0.110$ ) between cases and controls. At the time of biopsy, cases had significant lower mean eGFR (22.3 (SD +- 12.9)), compared to controls (mean eGFR 48.8 (SD +- 15.4; $\mathrm{p}<0.001$; Table 2)). Four weeks postbiopsy the mean eGFR of cases remained low with 25.7 (SD +- 16.1) whereas the mean eGFR of controls improved to 55.7 (SD +- 14.1) and there remained a significant difference between the eGFR of cases and controls 4 weeks post biopsy. $(p<0.001)$.

\section{Plasma cells and outcome}

Patients with prominent plasma cells had an increased rate of graft failure (Table 4). Graft failure with return to dialysis occurred in $10(71 \%)$ patients with plasma cells versus in one 
(7\%) without plasma cells $(p<0.001)$. One patient returned to PD, eight patients to HD and two patients (including the one from the control group) were re-transplanted.

Time to graft failure was 5 - 95 months (median 24) post transplantation, and $0-27$ months (median 2) post biopsy in the plasma cell group versus 53 months post transplantation and 14 months post biopsy in the patient without prominent plasma cells. (Figures 1 and 2).

From the plasma cell group, four patients had a functioning allograft until final follow up 3 23 months (median 12.5) post biopsy with a mean eGFR of $38.5( \pm 11.9)$. Three patients were transitioned to an adult unit with functioning allograft and one patient is still under ongoing care within our department.

From the controls, 13 patients had a functioning allograft until final follow up $2-75$ months (median 45) post biopsy with a mean eGFR of 51.7 ( \pm 20.8$)$. Nine were transitioned to an adult unit with functioning transplant and four are under ongoing care within our department.

Patient survival was $93 \%(13 / 14)$ for cases and $100 \%$ forcontrols. Death occurred in one case at three years and 11 months post transplantation and three years and six months post biopsy due to severe sepsis. The patient had a functioning allograft at time of death. 


\section{Discussion:}

With this retrospective case control study we have investigated the frequency and outcome of paediatric patients with plasma cell rich acute rejection, and have demonstrated that this complicates less than $10 \%$ of paediatric renal transplant recipients but is associated with a significantly increased risk of graft loss.

Plasma cell rich acute rejection is a relatively newly described entity and is characterized by the presence of prominent plasma cells as part of the interstitial inflammatory infiltrate.. These findings are rare but have been reported with a frequencyof $2-14 \%$ of adult cases with biopsy proven acute rejection (2) (4) (6) (7). In children there are only case reports reporting PCAR in renal transplant recipients (8). We studied paediatric transplant recipients who underwent an allograft biopsy for acute, chronic or acute on chronic allograft dysfunction and report a prevalence of $9 \%$, which is similar to results reported in the adult population.

Due to the low prevalence of PCAR it is difficult to definepossible risk factors. We did not identify any significant preceeding or concurrent events at the time of biopsy that differed from cases to controls. In particular, there were no significant differences in the baseline immunological criteria (type of transplant and number of HLA mismatches). Within the cases more patients were EBV positive at time of transplantation, but this was not a significant difference. Plasma cell infiltrates have been reported in association with EBV induced post transplantation lymphoproliferative diseases (PTLD) (3), but importantly, none of our patients with plasma cell infiltrates developed PTLD subsequently. Furthermore the plasma cell rich cases reported here were not atypical, and showed no other features of PTLD, wherefore a relation to EBV infection is very unlikely. None of the other clinical parameters, including age of the patient or age of transplant at time of biopsy were significantly different between both groups. In adult studies similar findings are reported (2) (5) (9). 
In our paediatric cohort we observed that at the time of biopsy patients with PCAR already had significantly reduced eGFR (mean of $22.3 \mathrm{vs} 48.8 \mathrm{mls} / \mathrm{min} / 1.73 \mathrm{~m}^{2}, \mathrm{p}<0.001$ ) compared to controls. Both groups received standard treatment for rejection according to BANFF classification. Four weeks post biopsy the patients with common acute rejections (CAR) showed an increase in mean eGFR to $55.6 \mathrm{mls} / \mathrm{min} / 1.73 \mathrm{~m}^{2}$, whereas the mean eGFR of cases remained significantly lower at $25.7 \mathrm{mls} / \mathrm{min} / 1.73 \mathrm{~m}^{2}(\mathrm{p}<0.001)$. Those findings confirm previously reported observations of poor response of PCAR to augmented immunosuppression in adult population (9).

The clinical significance and impact on graft survival has only been addressed in view adult studies and remains uncertain(2) (4) (5) (7). In one study of 16 patients with PCAR and 17 controls there was no significant difference in the proportion of grafts lost by one and three years of follow- up ( $50 \%$ vs $59 \%$ and $63 \%$ vs $76 \%, p=0.2)$ or median time from biopsy to graft loss (4.5 vs 6 months) between patients with PCAR and controls. In contrast, most of the other studies have reported poorer graft outcome and shorter time to graft loss in PCAR. For example graft loss of $52 \%$ in PCAR vs $20 \%(p<0.05)$ in classical acute rejections with a mean of 6.3 vs 21.5 months post biopsy (5) and overall 6 months graft survival rate after diagnosis of PCAR of $50 \%$ which was significantly lower controls (9). Similar findings with significantly more adverse prognosis of the group rich in plasma cells compared to those non rich in plasma cells are described elsewhere (7).

In our population, graft loss with return to dialysis occurred in $10(71 \%)$ patients with plasma cell infiltrates versus only one $(7.1 \%)$ patient without plasma cells $(p<0.001)$. Graft loss occurred also significantly earlier post biopsy in cases versus in controls (median two months vs 14 months). Furthermore, those patients with PCAR were more likely to develop more severe chronic allograft changes with lower eGFRs at final follow up. Similar findings are reported in adults, with serum creatinine remaining high in four out of five patients with PCAR and surviving graft in one study (9). Overall, data from the adult population and our current 
data strongly suggest that PCAR is associated with significantly worse outcome, independent of the severity of rejection according to other standard BANFF criteria.

In conclusion, the findings have demonstrated that the presence of plasma cell rich infiltrates in paediatric renal transplant biopsies is associated with reduced renal allograft survival and reduced allograft function in surviving grafts. National and international collaborative studies are required to investigate the incidence and significance of plasma cell infiltrates in paediatric renal transplant biopsies. In the future, targeted therapies in such cases may play a role in patient management. 
Table 1: Underlying diagnosis of patient population

\begin{tabular}{lll} 
Diagnosis & Cases & Controls \\
\hline CAKUT & 7 & 6 \\
FSGS & 1 & 2 \\
Juvenile Nephronophtisis & 2 & 1 \\
Cong NS & 1 & \\
HUS & & 1 \\
Bilateral Wilms tumour & & 1 \\
MPGN & 1 & \\
Bardet - Biedel & 1 & \\
St.p. heart lung TX & 1 & \\
Unknown aetiology & & 3 \\
\hline
\end{tabular}


Table 2: Characteristics of 14 patients with plasma cells in renal transplant biopsies in relation to 14 patients with no plasma cells in renal transplant biopsies

\begin{tabular}{|c|c|c|c|}
\hline & Cases $(n=14)$ & Controls $(n=14)$ & $\mathrm{p}$ - value \\
\hline \multicolumn{4}{|c|}{ Age of patient at biopsy (yrs) } \\
\hline Median & 15.9 & 13.5 & 0.21 \\
\hline Range & $4.8-17.9$ & $5.6-17.6$ & \\
\hline \multicolumn{4}{|c|}{ Age of graft at biopsy (months) } \\
\hline Median & 18.6 & 8.7 & 0.14 \\
\hline Range & $4.9-106.5$ & $0.7-103.2$ & \\
\hline \multicolumn{4}{|l|}{ Donor type } \\
\hline LRD & 1 & 5 & 0.165 \\
\hline $\mathrm{DD}$ & 13 & 9 & \\
\hline \multicolumn{4}{|l|}{ HLA mismatch } \\
\hline 0 antigen & 0 & 2 & \\
\hline 1 antigen & 4 & 0 & \\
\hline 2 antigen & 7 & 6 & \\
\hline 3 antigen & 1 & 4 & \\
\hline 4 antigen & 2 & 2 & \\
\hline \multicolumn{4}{|l|}{ Second transplant } \\
\hline Recipient EBV pos & 10 & 5 & 0.06 \\
\hline Recipient CMV pos & 3 & 6 & 0.23 \\
\hline \multicolumn{4}{|c|}{ Baseline eGFR (ml/min/ $1.73 \mathrm{~m})$} \\
\hline Mean & 75.9 & 88.2 & 0.175 \\
\hline SD & 19.9 & 26.7 & \\
\hline \multicolumn{4}{|c|}{ eGFR at biopspy (ml/min/ $1.73 \mathrm{~m})$} \\
\hline Mean & 22.3 & 48.8 & $<0.001$ \\
\hline $\mathrm{SD}$ & 12.9 & 15.4 & \\
\hline
\end{tabular}


Table 3: Grade of rejection according to BANFF classification

\begin{tabular}{lll} 
BANFF classification & Cases & Controls \\
\hline Acute rejection - Borderline & 2 & 3 \\
Acute rejection - I a & 3 & 3 \\
Acute rejection - I b & 2 & 1 \\
Acute rejection - II a & 3 & 3 \\
Acute rejection - II b & 1 & 1 \\
Chronic changes & 1 & 3 \\
Other & 2 & 0 \\
\hline
\end{tabular}


Table 4: Outcome

\begin{tabular}{clllll}
\hline & Cases & \multicolumn{5}{c}{ Controls } \\
\hline & Number & Percentage & Number & Percentage & P value \\
Functioning graft & 4 & $29 \%$ & 13 & $93 \%$ & $<0.001$ \\
Graft loss total & 10 & $71 \%$ & 1 & $7 \%$ & \\
$<1 \mathrm{mo}$ & 2 & & & & \\
$>1 \mathrm{mo}-1 \mathrm{yr}$ & 5 & & & & \\
$>1 \mathrm{yr}$ & 3 & & 1 & & \\
\hline
\end{tabular}


Figure 1: Kaplan-Meier plot of renal allograft survival at follow-up post-transplant

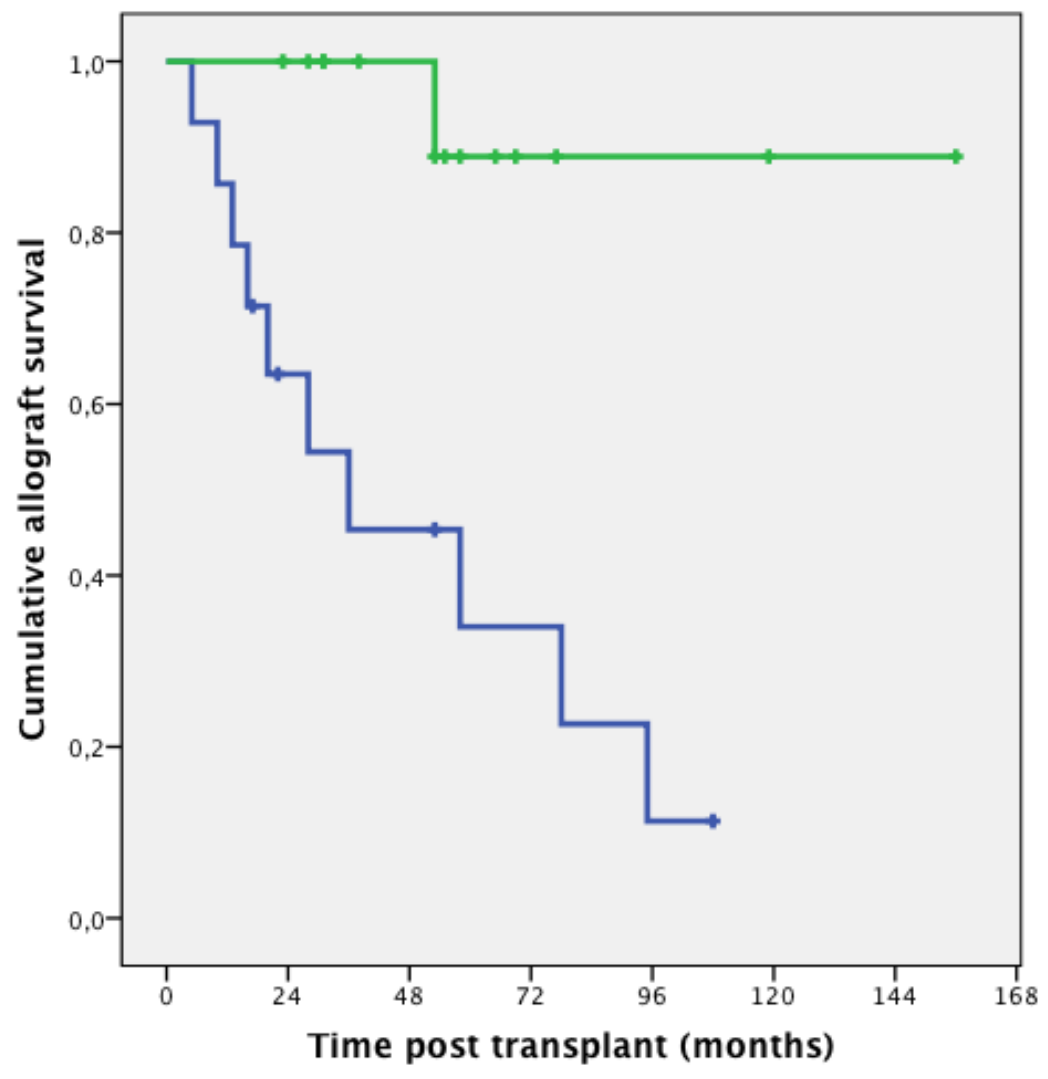


Figure 2 Kaplan-Meier plot of renal allograft survival at follow-up post-biopsy

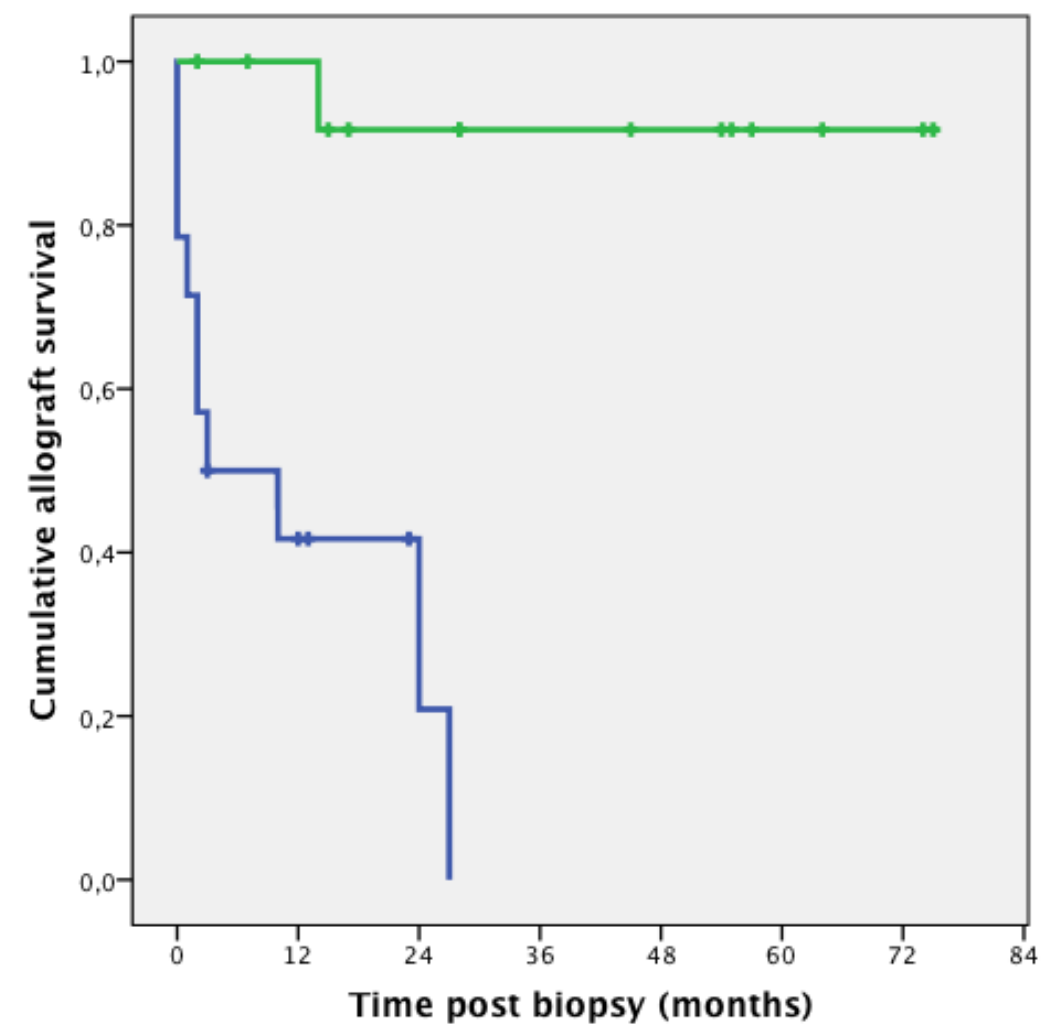




\section{References}

1. Racusen LC, Solez K, Colvin RB, Bonsib SM, Castro MC, Cavallo T, et al. The Banff 97 working classification of renal allograft pathology. Kidney international. 1999;55(2):713-23.

2. Desvaux D, Le Gouvello S, Pastural M, Abtahi M, Suberbielle C, Boeri N, et al. Acute renal allograft rejections with major interstitial oedema and plasma cell-rich infiltrates: high gamma-interferon expression and poor clinical outcome. Nephrology, dialysis, transplantation : official publication of the European Dialysis and Transplant Association - European Renal Association. 2004;19(4):933-9.

3. Sun IO, Cho YH, Hong YA, Chung BH, Choi BS, Park GS, et al. Plasma cell-rich acute rejection with monoclonal gammopathy in a renal transplant recipient. Experimental and clinical transplantation : official journal of the Middle East Society for Organ Transplantation. 2013;11(2):191-4.

4. Meehan SM, Domer P, Josephson M, Donoghue M, Sadhu A, Ho LT, et al. The clinical and pathologic implications of plasmacytic infiltrates in percutaneous renal allograft biopsies. Human pathology. 2001;32(2):205-15.

5. Charney DA, Nadasdy T, Lo AW, Racusen LC. Plasma cell-rich acute renal allograft rejection. Transplantation. 1999;68(6):791-7.

6. David-Neto E, Ribeiro DS, Ianhez LE, Palomino S, Saldanha LB, Arap S, et al. Acute interstitial nephritis of plasma cells: a new cause for renal allograft loss. Transplantation proceedings. 1993;25(1 Pt 2):897-9.

7. Gartner V, Eigentler TK, Viebahn R. Plasma cell-rich rejection processes in renal transplantation: morphology and prognostic relevance. Transplantation. 2006;81(7):986-91.

8. Chikamoto H, Sugawara N, Akioka Y, Shimizu T, Horita S, Honda K, et al. Immunohistological study of a pediatric patient with plasma cell-rich acute rejection. Clinical transplantation. 2012;26 Suppl 24:54-7.

9. Gupta R, Sharma A, Mahanta PJ, Agarwal SK, Dinda AK. Plasma cell-rich acute rejection of the renal allograft: A distinctive morphologic form of acute rejection? Indian journal of nephrology. 2012;22(3):184-8. 